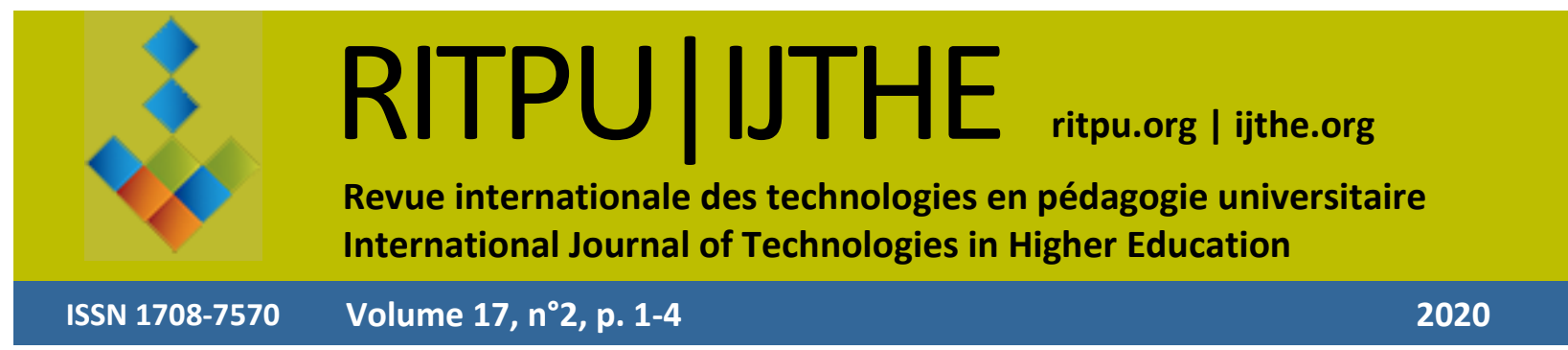

\title{
Le numérique et l'enseignement au temps de la COVID-19 : entre défis et perspectives - Partie 1
}

https://doi.org/10.18162/ritpu-2020-v17n2-01

Thierry KARSENTI

thierry.karsenti@umontreal.ca

Bruno POELLHUBER

bruno.poellhuber@umontreal.ca

Normand ROY

normand.roy@umontreal.ca

Simon PARENT

simon.parent.2@umontreal.ca

Université de Montréal

Canada

\section{Mis en ligne : 23 novembre 2020}

En raison de la situation mondiale liée à la COVID-19, et selon les données publiées par l'UNESCO (2020a), quelque 1,6 milliard d'apprenants dans le monde, du préscolaire à l'université, n'ont pas été en mesure, à un moment ou un autre, de fréquenter leur établissement d'enseignement depuis le début de la pandémie. Concrètement, ce chiffre inquiétant confirme que plus de $94 \%$ des apprenants scolarisés sur Terre ont été touchés par cette crise sanitaire. À ce jour, plus de 190 pays ont ordonné la fermeture de l'ensemble de leurs établissements d'enseignement, des écoles primaires aux universités, à un moment ou un autre, au cours des derniers mois. Et avec l'arrivée de la «deuxième vague » de COVID-19, les établissements d'enseignement universitaires se sont une fois de plus dirigés vers l'enseignement à distance, et ce, pour plusieurs mois.

Les termes utilisés pour nommer cette crise éducative (continuité pédagogique, mesures alternatives, enseignement ou apprentissage à la maison, etc.) témoignent de l'empressement à implanter une approche transposant les habitudes de l'enseignement du présentiel au distanciel. Et bien que plusieurs hésitent à l'admettre, nous entrons de plain-pied dans le domaine de la formation à distance, avec ses avantages (notamment la flexibilité), mais aussi ses défis pour les apprenants (motivation, engagement et persévérance), pour les enseignants (conception des cours, encadrement des apprenants) et pour les établissements d'enseignement postsecondaire, dont plusieurs n'ont aucune expérience dans l'enseignement en ligne.

Dans la situation actuelle où la formation à distance est à l'avant-plan des préoccupations éducatives des établissements d'enseignement, et ce, résolument pour les prochains mois, voire pour les prochaines années, il a semblé opportun de rassembler les expertises de recherche et les expertises pédagogiques sur les différentes manières de faire face aux nombreux problèmes que pose la pandémie pour les universités. C'est donc dans le cadre de cette crise sanitaire planétaire que nous avons lancé, en juin 2020, un appel à textes pour ce numéro thématique publié dans la 
Revue internationale des technologies en pédagogie universitaire (RITPU). Alors que la Revue publie habituellement de 15 à 20 textes par année, les quelque 110 textes reçus constituaient un record absolu pour un seul appel. C'est dans ce contexte que nous avons lancé ce numéro dont les textes seront publiés de façon continue, conformément aux politiques de la Revue, au cours des prochaines semaines, voire des prochains mois. Tous les textes réunis, soumis au rigoureux processus d'évaluation par les pairs, portent évidemment sur l'enseignement ou l'apprentissage au postsecondaire. Et bien que, de façon générale, la Revue publie davantage des recherches scientifiques avec données empiriques, sa politique éditoriale permet également la publication de comptes rendus d'expériences, si ces dernières sont ancrées dans la littérature scientifique. Il s'avère que dans le contexte actuel, nous avons jugé important d'accorder une place particulière à ce type de publications qui permettront de faire un tour d'horizon des mesures prises à travers le monde en formation à distance au postsecondaire.

Ce numéro thématique, tout comme ceux qui suivront et qui présenteront les travaux de chercheurs qui se sont intéressés à ce contexte particulier et au numérique en éducation, semble particulièrement important aujourd'hui. En effet, il nous parait impératif de documenter, par la recherche, par des retours d'expériences ancrés dans la recherche de même que par des perspectives théoriques, la mise en place de diverses solutions pour faire face à cette crise éducative exceptionnelle, surtout parce qu'elle n'est pas terminée. Cette mutualisation des connaissances est essentielle pour éclairer de meilleures prises de décision. Les textes retenus proviennent de quelque 30 pays des quatre coins du monde comme le Brésil, la Belgique, le Canada, la Côte d'Ivoire, les États-Unis, la France, le Sénégal... et même le royaume d'Eswatini, petit pays d'Afrique australe. Ces travaux, présentés par des chercheurs de partout sur Terre, attestent, entre autres, de plusieurs des perturbations sismiques vécues dans les universités depuis le début de cette crise. Les enjeux présentés dans les textes de ce numéro thématique sont significatifs, tant pour les apprenants que pour les formateurs et les gestionnaires d'établissement, et même pour les États. Et, selon les nombreuses recherches et les divers récits d'expérience présentés, ces enjeux ont manifestement amené les établissements d'enseignement à se transformer en quelques mois, comme le soulignait d'ailleurs Witze (2020), tout récemment, dans un texte publié dans Nature. En fait, comme le montrent les textes de ce numéro thématique, plusieurs universités ont été contraintes de réinventer l'enseignement supérieur.

Les défis en pédagogie universitaire relevés au cours de la pandémie et abordés dans ce numéro exceptionnel de la RITPU, et dans ceux qui suivront, sont nombreux. On y retrouve évidemment, sous diverses formes, le passage de l'enseignement en présentiel à l'enseignement en ligne, devenu une préoccupation centrale pour les universités du monde entier qui semblent s'être métamorphosées en quelques mois, grâce à des efforts herculéens au cours de l'été dernier, pour préparer leurs formateurs. C'est d'ailleurs ce que montre la dernière enquête d'EDUCAUSE où l'on apprend que les universités ont consacré la majeure partie de leur été à améliorer leurs pratiques de formation à distance de même qu'à perfectionner leur usage d'outils numériques destinés à l'enseignement en ligne (voir McCormack, 2020). Les auteurs des recherches présentées dans ce numéro thématique ont voulu non seulement établir les avantages de ce type de formule pédagogique, mais aussi, et peut-être surtout, documenter les écueils auxquels sont confrontés apprenants, formateurs et autres acteurs éducatifs impliqués dans cette nouvelle réalité pédagogique de l'enseignement postsecondaire à l'ère de la COVID-19. C'est le cas notamment de L'efficacité d'un dispositif d'enseignement hybride en fonction des caractéristiques des étudiants ( $1^{\mathrm{er}}$ texte), de Enseigner le travail social en contexte de pandémie $\therefore$ un exemple d'environnement d'apprentissage hybride en ligne sur Moodle ( $3^{\mathrm{e}}$ texte), COVID19 et continuité pédagogique en licence 3 , quelles leçons pour demain? ( $4^{\mathrm{e}}$ texte), Creating and 
Teaching an Online Business Case Study using Twine (5 $5^{\mathrm{e}}$ texte), Navigating a "New Normal" during the COVID-19 Pandemic: College Student Perspectives of the Shift to Remote Learning ( $6^{\mathrm{e}}$ texte), Expérimentation d'une séance de simulation managériale en classe inversée sans regroupement physique ( $7^{\mathrm{e}}$ texte), Going remote: Faculty educational initiatives on building community during COVID-19 through online teaching ( $8^{\mathrm{e}}$ texte), Repenser l'expérience d'enseignement et d'apprentissage en situation de confinement pédagogique $\left(11^{\mathrm{e}}\right.$ texte), The Hyber-Flexible Course Design Model (HyFlex): A Pedagogical Strategy for Uncertain Times (12 $2^{\mathrm{e}}$ texte).

On y aborde également les questions d'équité, devenues un enjeu majeur pour les universités et les États. En effet, comme le montrent les travaux de Karsenti et al. (2020) ou le rapport de l'International Association of Universities (2020), la pandémie a exacerbé encore davantage les iniquités sociales existantes, tant dans les établissements d'enseignement que sur le plan de l'usage du numérique. Concrètement, les apprenants issus des milieux les plus démunis semblent avoir rencontré des difficultés particulières, notamment en ce qui a trait à l'accès aux appareils numériques et à Internet. En effet, on observe ces problèmes d'équité non seulement entre les apprenants d'un même établissement d'enseignement, mais de façon encore plus marquée entre les apprenants de différents pays. Par exemple, comme l'indiquait l'UNESCO (2020b), la COVID-19 a confirmé une fracture numérique très préoccupante dans la formation à distance, tout particulièrement en Afrique où $82 \%$ des apprenants n'ont pas accès à Internet à domicile. Dans ce numéro thématique particulier, cette question est abordée dans Navigating a "New Normal" during the COVID-19 Pandemic: College Student Perspectives of the Shift to Remote Learning ( $6^{\mathrm{e}}$ texte), mais surtout dans Enseignement à distance et inégalités au Mexique : les limites de la continuité éducative ( $9^{\mathrm{e}}$ texte).

Ce numéro thématique traite évidemment de la préparation des formateurs à l'enseignement en ligne (voir Crawford et al., 2020), comme c'est le cas par exemple pour Conception d'une nouvelle unité de formation à l'enseignement en mode COVID 19 - Doutes, incertitudes et nouveaux apprentissages $\left(10^{\mathrm{e}}\right.$ texte). La question de la réussite éducative est une préoccupation fort importante, non seulement pour les étudiants universitaires eux-mêmes (Karsenti et al., sous presse), mais également pour les gouvernements qui se demandent souvent, à ce stade de la crise sanitaire, quelles retombées, susceptibles d'affecter directement les apprenants, sont le plus à craindre (International Association of Universities, 2020), ce sont notamment certaines des questions traitées dans COVID-19 et continuité pédagogique en licence 3, quelles leçons pour demain? ( $4^{\mathrm{e}}$ texte). Sont également traitées les questions de l'évaluation en ligne, complexe dans un tel contexte de pandémie, toujours selon l'IAU, et celle du soutien aux étudiants, à distance, qui représente un défi de taille (Sahu, 2020), thématique abordée dans Évaluation de fonctionnaires-stagiaires à distance - Un exemple à l'Inspé de l'académie de Limoges $\left(13^{\mathrm{e}}\right.$ texte). Finalement, la question de l'adaptation à cette nouvelle normalité, pour les étudiants universitaires, en particulier si la crise devait perdurer (Brammer et Clark, 2020), est aussi examinée, comme c'est le cas par exemple dans Pédagogie universitaire et numérique : le défi d'une éthique de l'attention ( $2 \mathrm{e}$ texte).

Cette situation surréelle a amené les universités et les États à prioriser deux missions : la première est de trouver une parade à la crise sanitaire qui sévit; la seconde, et c'est là que ce numéro thématique vient jouer un rôle-clé, est de surmonter la crise des apprentissages en enseignement supérieur pour réduire au maximum les effets d'une «génération COVID ». En effet, les textes présentés sont un premier pas pour tenter à la fois de baliser, de mieux comprendre et de trouver des pistes de solution à ces défis en enseignement supérieur posés par 
la pandémie de COVID-19. Les leçons tirées de ces études et récits d'expérience divers pourront à la fois aider apprenants, formateurs et établissements à braver les nombreux défis rencontrés et contribuer à les rassurer devant l'inconnu.

\section{Références}

Brammer, S. et Clark, T. (2020). COVID-19 and management education: Reflections on challenges, opportunities, and potential futures. British Journal of Management, 31(3), 453-456. https://doi.org/10.1111/1467-8551.12425

Crawford, J., Butler-Henderson, K., Rudolph, J., Malkawi, B., Glowatz, M., Burton, R., Magni, P. A. et Lam, S. (2020). COVID-19: 20 countries' higher education intra-period digital pedagogy responses. Journal of Applied Learning \& Teaching, 3(1), 9-28. https://doi.org/10.37074/jalt.2020.3.1.7

International Association of Universities. (2020). COVID-19: Higher education challenges and responses. http://iau-aiu.net/...

Karsenti, T., Parent, S. et Cuerrier, M. (2020). L'école à la maison : la pandémie a-t-elle réellement exacerbé les iniquités sociales? Éducation Canada, 60(4), 4-9.

Karsenti, T., Parent, S., Cuerrier, M. et Ranger, F. (sous presse). Défis, avantages et stratégies gagnantes en contexte de pandémie : résultats d'une étude auprès de 4179 étudiants universitaires. Formation et profession.

McCormack, M. (2020, 7 août). EDUCAUSE QuickPoll results: Fall planning for online and physical spaces. EDUCAUSE Review. http://er.educause.edu/blogs/...

Sahu, P. (2020). Closure of universities due to coronavirus disease 2019 (COVID-19): Impact on education and mental health of students and academic staff. Cureus, 12(4). Article e7541. https://doi.org/10.7759/cureus.7541

UNESCO. (2020a). COVID-19 : une crise mondiale pour l'enseignement et l'apprentissage. http://unesdoc.unesco.org/...

UNESCO. (2020b, 5 août). UN Secretary-General warns of education catastrophe, pointing to UNESCO estimate of 24 million learners at risk of dropping out [communiqué de presse]. http://en.unesco.org/...

Witze, A. (2020, $1^{\text {er }}$ juin). Universities will never be the same after coronavirus crisis. Nature, 582, 162-164. https://doi.org/10.1038/d41586-020-01518-y 